\title{
Optical orbital angular momentum: twisted light and chirality
}

\author{
Kayn A. Forbes And David L. Andrews* \\ School of Chemistry, University of East Anglia, Norwich NR4 7TJ, United Kingdom \\ *Corresponding author: david.andrews@physics.org
}

Received XX Month XXXX; revised XX Month, XXXX; accepted XX Month XXXX; posted XXX MOnth XXXXX (DOC. ID XXXXX); published XXX Month XXXX

The question of how the orbital angular momentum of structured light might engage with chiral matter is a topic of resurgent interest. By taking account of electric quadrupole transition moments, it is shown that the handedness of the beam can indeed be exhibited in local chiral effects, being dependent on the sign of the topological charge. In the specific case of absorption, a significant interplay of wavefront structure and polarization is resolved, and clear differences in behavior are identified for systems possessing a degree of orientational order and for those that are randomly oriented.

OCIS codes: (020.0020) Atomic and molecular physics; (270.5580) Quantum electrodynamics; (160.1585) Chiral media; (080.4865) Optical vortices

http://dx.doi.org/10.1364/0L.99.099999

It is now well established that photons can possess two essentially different forms of angular momentum: spin and orbital [1]. In the paraxial regime, the spin angular momentum (SAM) manifest in circular polarization is restricted to helicity eigenvalues of $\pm \hbar$ per photon. However, the orbital angular momentum (OAM) associated with many forms of structured light has no theoretical bounds on dimensionality [2]. In 'twisted' beams each photon can possess OAM values of $\pm \ell \hbar$, where $\ell$ is an integer representing the topological charge that characterizes the wavefront structure.

The question of how such an orbital angular momentum of structured light might engage with chiral matter is a topic of resurgent interest - see for example [3-5]. Chiral interactions and discriminatory effects have long been associated with the differential response of circularly polarized photons, in their interactions with right or left-handed chiral molecules [6-8]. Archetype examples include circular dichroism [9], optical rotation [10] and differential scattering [11], supplemented by more recent developments in optical trapping [12] and binding forces [13]. The mechanisms responsible for these discriminatory optical processes and phenomena generally involve a coupling between electric dipole (E1) and either magnetic dipole (M1) or electric quadrupole
(E2) transition moments. The origin of such interactions can be succinctly explained in terms of the overall $P T$ symmetry afforded by the underlying electrodynamics, associated with the fact that E1 moments have odd spatial parity, and M1 and E2 even parity. The E1-M1 and E1-E2 interference terms therefore change sign on spatial inversion - and in consequence differ for left- and righthanded forms of any chiral molecule.[8,11]

Light propagating with any form of helical wavefront is inherently chiral, the beam twisting to the right for $\ell<0$ and to the left for $\ell>0$. It is for this reason that one would anticipate, in chiral matter, discriminatory effects with respect to the orbital angular momentum: a beam with positive $\ell$ should produce a different effect from one with negative $\ell$. Studies on OAM and chirality in light-matter interactions have previously focused on E1 and M1 couplings, indicating that wavefront handedness should play no part in chiral discrimination, and only circular polarization (SAM) could lead to any differential effects [3]. This was later experimentally verified $[14,15]$.

In considering the possibility of electric quadrupole effects, it is interesting to first reflect on the issue of physical scale with regard to optical vortices. It may be borne in mind that electric quadrupole effects can contribute to the exhibition of conventional molecular chirality - as for example in circular differential Rayleigh and Raman scattering [11]. At a given radial and angular displacement from the beam axis, the gap between successive wavefronts in an optical vortex is the same wavelength as for non-vortex light (it just intercepts a different member of the set of $\ell$ individual helicoidal wavefronts).

It is shown in this paper that due consideration of electric quadrupole (E2) moments does indeed reveal a basis for the chirality associated with photon OAM to produce chiral effects in light-matter interactions, such that the rates of optical processes can in fact depend on the direction in which the vortex beam is twisting, and therefore the sign of $\ell$. Moreover the results explicitly depend on the magnitude of $\ell$, which quantifies the orbital angular momentum.

In the work presented we adopt the Power-Zienau-Woolley (PZW) Hamiltonian to study the interplay between radiation and 
material [16]. In the PZW representation, the Hamiltonian for the interaction between matter and quantized radiation is expanded in terms of multipole moments and their engagement with the transverse electromagnetic field:

$$
\begin{aligned}
H_{\mathrm{int}}= & -\sum_{\xi} \mu_{i}(\xi) e_{i}^{\perp}\left(\boldsymbol{R}_{\xi}\right)-\sum_{\xi} Q_{i j}(\xi) \nabla_{j} e_{i}^{\perp}\left(\boldsymbol{R}_{\xi}\right)- \\
& \cdots-\sum_{\xi} m_{i}(\xi) b_{i}\left(\boldsymbol{R}_{\xi}\right)-\cdots
\end{aligned}
$$

where there is an implied summation over subscript component indices. In (1), the first term involves the electric dipole $\boldsymbol{\mu}$ coupling, (E1); the second term involves the electric quadrupole $\boldsymbol{Q}$ (E2); and the final term is the magnetic dipole $\boldsymbol{m}$ (M1). To correctly account for the electromagnetic field being able to possess an orbital angular momentum of $\pm \ell \hbar$ per photon, the electromagnetic field needs to be cast in a form that accommodates an azimuthal phase factor $\exp (i \ell \phi)[17,18]$. The most widely utilized solutions to the wave equation in the paraxial approximation are LaguerreGaussian (LG) modes, whose radial profile is cast as a Gaussian distribution modified by one of the associated (generalized) Laguerre polynomials. The rotational symmetry of such beams invites casting the transverse field operators $\boldsymbol{e}^{\perp}$ and $\boldsymbol{b}$ in (1) in terms of cylindrical coordinates [19].

Looking in particular at the coupling between E2 and E1 multipoles, it will now be shown that it is possible exhibit chiral discrimination with regards to the sign of the orbital angular momentum, assuming paraxial beam propagation. To highlight and exemplify our general findings, we choose the most fundamental of optical processes: one-photon absorption. To continue, we can study the time evolution of the system wavefunction using timedependent perturbation theory. The matrix element including only the E1 and E2 couplings is thus seen to be:

$$
\begin{aligned}
M_{f i} & =\left\langle\text { final }\left|H_{\mathrm{int}}\right| \text { initial }\right\rangle \\
& =-\left\langle E_{m}\left|\mu_{i}\right| E_{o}\right\rangle\left\langle(n-1)\left|e_{i}^{\perp}(\boldsymbol{r})\right| n\right\rangle \\
& -\left\langle E_{m}\left|Q_{i j}\right| E_{o}\right\rangle\left\langle(n-1)\left|\nabla_{j} e_{i}^{\perp}(\boldsymbol{r})\right| n\right\rangle,
\end{aligned}
$$

where $|E\rangle$ designate molecular states and $|n\rangle$ is the radiation state belonging to a specific mode $(\boldsymbol{k}, \eta, \ell, p)$. Here, $\boldsymbol{k}$ is the wave-vector, $\eta$ a polarization label, $\ell$ is the topological charge - which for an LG beam equates to the degree of the Laguerre polynomial, and $p$ is the corresponding order. Expanding the Dirac brackets in (2) using the transverse electric field expansion for LG beams, the matrix element (2) can be written as

$$
M_{f i}=-i\left(\frac{n \hbar \omega}{2 \varepsilon_{0} V}\right)^{1 / 2} \varepsilon_{i}\left(\mu_{i}^{m o}+Q_{i j}^{m o} \nabla_{j}\right) f_{\ell, p}(r) \mathrm{e}^{(i k z+i \ell \phi)}
$$

in which $\varepsilon_{i}$ is the unit polarization vector, $n$ is the number of photons in the quantization volume $V$, and $f_{\ell, p}(r)$ is the appropriately normalized radial distribution function. The second term in brackets in (3), which involves the coupling of electric quadrupole moment components with the gradient of the transverse electric field, requires specific attention. As mentioned above, the mode expansions for the electromagnetic field may be cast in a cylindrical coordinate system $(z, \phi, r)$, and $\nabla$ in (3) operates accordingly. The crux of the result emerges from $\nabla_{j} f_{\ell, p}(r) \mathrm{e}^{(i k z+i \ell \phi)}$, whose implementation leads to:

$$
\begin{aligned}
M_{f i}^{\prime} & =-i\left(\frac{n \hbar \omega}{2 \varepsilon_{0} V}\right)^{1 / 2} f_{\ell, p}(r) \varepsilon_{i} \\
& {\left[\mu_{i}^{m o}+r^{-1} Q_{i j}^{m o}\left(i \ell \hat{\phi}_{j}-\hat{r}_{j}\right)\right] \mathrm{e}^{(i k z+i \ell \phi)}, }
\end{aligned}
$$

where the prime symbol on the left signifies removal of terms that, in the ensuing rate equation, cannot contribute to an overall dependence on the sign of $\ell$. This removal of terms is made more clear in the correct anticipation that for any parametric or nonparametric optical processes, once the rate is calculated through the Fermi rate rule, all information on wave-front handedness through the sign of $\ell$ in the phase factor $\exp (i \ell \phi)$ is lost in the ensuing modulus square of the amplitude. Using the aforementioned Fermi rule, we now obtain for the rate:

$$
\Gamma^{\prime}=\frac{I(\omega) N}{2 c \hbar^{2} \varepsilon_{0}} f_{\ell, p}^{2}(r) \varepsilon_{i} \bar{\varepsilon}_{k}\left|\mu_{i}^{m o}+r^{-1} Q_{i j}^{m o}\left(i \ell \hat{\phi}_{j}-\hat{r}_{j}\right)\right|^{2},
$$

where $I(\omega)$ is the irradiance per unit frequency interval, which when integrated over frequency represents the intensity of the input beam, and $N$ is the number of absorbers. Once again retaining only those terms that are dependent on the sign of $\ell$, securing the final modulus square of (5) gives:

$$
\begin{aligned}
& i \ell\left(r^{-1} \hat{\phi}_{j} \mu_{k}^{m o} Q_{i j}^{m o}-r^{-1} \hat{\phi}_{l} \mu_{i}^{m o} Q_{k l}^{m o}\right. \\
& \left.+r^{-2} \hat{r}_{j} \hat{\phi}_{l} Q_{i j}^{m o} Q_{k l}^{m o}-r^{-2} \hat{\phi}_{j} \hat{r}_{l} Q_{i j}^{m o} Q_{k l}^{m o}\right) .
\end{aligned}
$$

Therefore it can be seen that we produce a mixture of E1-E2 and E2-E2 terms that are all dependent on the optical orbital angular momentum, as determined by the sign and magnitude of $\ell$. However, we can neglect the E2-E2 terms as they are insignificant in magnitude compared to the E1-E2 terms - although they may have a capacity, in achiral media, to generate discriminatory effects with regard to the relative handedness of the spin and orbital angular momentum. This then leaves us with a vortex-modified component of the one-photon absorption rate, signified below by $\Gamma^{\prime \prime}$, that contains only the leading-order E1-E2 terms dependent on the sign of $\ell$ as:

$$
\Gamma^{\prime \prime}=\frac{I(\omega) N}{2 c \hbar^{2} \varepsilon_{0}} f_{\ell, p}^{2}(r) r^{-1} \varepsilon_{i} \bar{\varepsilon}_{k} \hat{\phi}_{j} i \ell\left(\mu_{k}^{m o} Q_{i j}^{m o}-\mu_{i}^{m o} Q_{k j}^{m o}\right),
$$

Evidently - since all of the other parameters in the above equation are real - the polarization vectors must be complex to produce a non-zero real result. At this stage we can therefore conclude that a vortex beam comprised of plane polarized photons will show no chiral effects in one-photon absorption - but that if the photons are 
circularly polarized, we can expect a chiroptical response. That is to say, at the most significant order - E1-E2 - the orbital angular momentum cannot produce chiroptical effects alone, but only in conjunction spin angular momentum. In this sense, the process is seen to be an analogue of circular dichroism (CD) but with beams carrying a topological charge - circular-vortex dichroism (CVD). It is worth pointing out, however, that discriminatory effects involving linear polarization states of light are possible when molecular response is modified, e.g. through plasmonic resonance, a process that has been called 'helical dichroism' .[5] We do not rule out the possibility of other terms allowing a dichroism depending only on a twisted wavefront, but they do not arise in the leading level of multipolar interaction, and in the paraxial approximation, as examined here.

To proceed further we now make use of the following identities [8]:

$$
\begin{aligned}
\varepsilon_{i}^{\mathrm{L}} \bar{\varepsilon}_{k}^{\mathrm{L}} & =\frac{1}{2}\left[\left(\delta_{i k}-\hat{k}_{i} \hat{k}_{k}\right)-i \varepsilon_{i k m} \hat{k}_{m}\right], \\
\varepsilon_{i}^{\mathrm{R}} \bar{\varepsilon}_{k}^{\mathrm{R}} & =\frac{1}{2}\left[\left(\delta_{i k}-\hat{k}_{i} \hat{k}_{k}\right)+i \varepsilon_{i k m} \hat{k}_{m}\right] .
\end{aligned}
$$

which allow us to write the final result as:

$$
\begin{aligned}
\Gamma^{(\mathrm{L})}-\Gamma^{(\mathrm{R})} & =\Delta \Gamma_{\mathrm{CD}}+\frac{I(\omega) N}{2 c \hbar^{2} \varepsilon_{0}} f_{\ell, p}^{2}(r) r^{-1} \\
& \times \varepsilon_{i k m} \hat{k}_{m} \hat{\phi}_{j} \ell\left(\mu_{k}^{m o} Q_{i j}^{m o}+\mu_{i}^{m o} Q_{k j}^{m o}\right) .
\end{aligned}
$$

It is to be emphasized that the result is cast in terms of the absolute difference in absorption rates between left- and right-handed circularly polarized photons. Here $\Delta \Gamma_{\mathrm{CD}}$ represents all of the terms that have no dependence on the sign of the topological charge. It is important to retain these terms in the final result as, when $\ell=0$, the total rate reduces to the standard result for $C D$, in precise agreement with the expression given in ref. [8] for beams without orbital angular momentum. It is also worth noting that the magnitude of the CD and CVD contributions will in general be similar (when all E1, M1 and E2 couplings are accounted for, not just terms dependent on the sign of the topological charge), since E2 and M1 interactions with the field are approximately equal in strength the CVD result will, nonetheless, scale linearly with $\ell$.

Now it is worth concentrating on the separate factors in (9). An important facet of the result emerges; the CVD differential (in these leading terms) is linearly dependent on the three aspects of handedness in the complete matter-radiation system - which are: the sense of circular polarization, which we can denote by $\eta= \pm 1$ for left/right-handed polarization; sign of the topological charge $\ell$; molecular chirality. For the last of these, it is clear that the product $\mu-Q$ is odd with respect to the parity operator $P$, and therefore it too will change sign upon inversion - this is the equivalent of changing the L/R handedness of either enantiomer; see Table 1 . Thus we can have eight distinct possible ways to permute the system, which can only lead to two different outcomes. Since we are specifically focusing on the differential absorption on changing left to righthanded circularly polarized input, there are four possible combinations: using the designation ' $\ell$ mol', the possible arrangements are $\mathrm{RR} \equiv \mathrm{LL} \neq \mathrm{LR} \equiv \mathrm{RL}$. Thus, for example, the CVD component of the dichroic response, defined by association with changing from left to right-handed circular polarization, will be the same for a vortex beam of right-handed wavefront structure interacting with one enantiomer as for the corresponding lefthanded vortex engaging with the opposite enantiomer; additionally, the standard $\mathrm{CD}$, independent of the topological charge, will still of course persist.

Table 1: Spatial parities of the key constituents in the CVD.

\begin{tabular}{ccc}
\hline Handedness & Parameter & $\begin{array}{c}\text { Parity } \\
\text { signature }\end{array}$ \\
\hline$\eta$ & $\varepsilon_{i k m} \hat{k}_{m}$ & +1 \\
$\ell$ & $\ell \hat{\phi}_{j}$ & -1 \\
mol & $\mu_{i}^{m o} Q_{k j}^{m o}-\mu_{k}^{m o} Q_{i j}^{m o}$ & -1 \\
\multicolumn{2}{c}{ The parameter $\boldsymbol{\eta}$ designating a circular polarization difference } \\
has even parity, since unit wave-vector $\hat{\boldsymbol{k}}$ contracts with the \\
Levi-Civita tensor, which is a pseudoscalar.
\end{tabular}

As it stands, the result (9) applies to one or more molecules with a fixed orientation at any point in the LG beam. Let us now focus on the presence of the $\widehat{\phi}_{j}$ term. Since this factor contracts with an index of the quadrupole transition moment - which itself has a fixed orientation within the molecule - the magnitude and sign of the CVD differential will in general vary around the beam axis. Specifically, the CVD will vary between a maximum and minimum of opposite sign, across the beam profile. This effect registers the different directions of phase gradient around each intensity ring, as experienced by chiral molecules with a common orientation. This in turn means that in a system of molecules that possess a degree of orientational order, such as a poled liquid crystal, the observed differential $\Gamma^{(\mathrm{L})}-\Gamma^{(\mathrm{R})}$ will be enhanced on one side of the beam, and diminished on the other - as compared with the standard CD value along the singular core. To fully verify the mechanism, a potential method is to conduct experiments with varying values of $\ell$, plotting against it the results for $\Gamma^{(\mathrm{L})}-\Gamma^{(\mathrm{R})}$, probing the locally differential absorption whilst taking account of the differences in intensity distribution associated with various radial distribution functions $f_{\ell, p}(r)$. Such experiments would appear to necessitate resolving the extent of absorption at different locations within the beam profile.

To complete the analysis, we now consider the consequences of a lack of orientational order. In contrast to liquid crystals, most molecular fluids have randomly oriented molecules, and there are seldom any effective means for introducing orientational order. To discover the effect of random orientation we therefore have to perform an isotropic rotational average of the expression for CVD. This requires the E1-E2 and E2-E1 terms to be contracted with the corresponding third rank isotropic tensor, namely the Levi-Civita epsilon [20]. However, since the electric quadrupole moment is symmetric in its indices, and the Levi-Civita fully index antisymmetric, the resulting molecular average is zero. Thus, it can be concluded that the extent of differential one-photon absorption, with a circularly polarized vortex beam, the CVD is non-zero for oriented systems but it vanishes for randomly oriented molecules. 
This net zero contribution to CVD in systems lacking orientational order is consistent with the null results of previous studies [14].

It is to be stressed that the results presented here provide a broader basis for further study in the area of twisted light beams and their potential significance in chiral light-matter interactions. The identification of mechanism, and explicit proof, of how a vortex structure can exert an influence on circular dichroism, gives a strong indication of how such a feature might also be manifest in other forms of chiroptical behaviour. The inclusion of electric quadrupole moments of matter in conjunction with structured light beams can be anticipated to lead to other discriminatory effects with a dependence on the sign of the topological charge. Notably, for example, recent experiments suggest a significant role in circular differential Raman scattering - see [21].

A further consideration is the possibility of engineering special systems for chiral effects using twisted light, which could serve a similar purpose to those observed in nonlinear optics [22], and would allow normally forbidden transitions and processes to have non-zero amplitudes. Particular examples involve the exploitation of the degree of molecular ordering that is present at boundaries of isotropic systems [23], introducing molecular alignment using optical methods [24], or applying a magnetic field to induce symmetry breaking [25]. It can be appreciated that in conventional systems, and 'normal' beams of light, E2 moments usually generate small contributions to the overall amplitude of an optical process, but for vortex beams the gradient of the electromagnetic fields clearly becomes of increasing significance as the topological charge increases. Indeed, this is manifest in the linear dependence on $\ell$ in equation (9). The enhanced role of such interactions is already recognized for light with OAM interacting with atoms $[26,27]$, and the study of enhancing their magnitude in light-matter interactions is an engaging field of research $[28,29]$.

To conclude it has been shown that, through the engagement of electric quadrupole interactions, the optical orbital angular momentum of light can exert a significant chiroptical influence in light-matter interactions, with the sign and magnitude of the topological charge influencing the local rate of one-photon absorption in oriented chiral systems. It is hoped that this work will now stimulate further study for other optical processes and experimental observations, where the possibility of varying the topological charge could lead to potentially significant advances in the burgeoning field of optical forces and nanomanipulation [30], chiral detection and chiroptical spectroscopy [31], and enantiomer separation $[32,33]$, to name but a few.

References

1. D. L. Andrews and M. Babiker, The Angular Momentum of Light (Cambridge University Press, Cambridge, UK, 2013).

2. G. Gibson, J. Courtial, M. J. Padgett, M. Vasnetsov, V. Pas'ko, S. M. Barnett, and S. Franke-Arnold, Opt. Express 12, 5448 (2004).

3. D. L. Andrews, L. C. Dávila Romero, and M. Babiker, Opt. Commun. 237, 133 (2004).

4. X. Zambrana-Puyalto, X. Vidal, and G. Molina-Terriza, Nat. Commun. 5, 4922 (2014).

5. W. Brullot, M. K. Vanbel, T. Swusten, and T. Verbiest, Science Advances 2, e1501349 (2016).

6. D. J. Caldwell and H. Eyring, The Theory of Optical Activity (Wiley-Interscience, New York, 1971).
7. S. F. Mason, Molecular optical activity and the chiral discriminations (Cambridge University Press, 1982).

8. D. P. Craig and T. Thirunamachandran, Molecular Quantum Electrodynamics: An Introduction to Radiation-Molecule Interactions (Dover Publications, Mineola, NY, 1998).

9. M. Nieto-Vesperinas, Phil. Trans. R. Soc. A 375 , 20160314 (2017).

10. P. L. Polavarapu, Chirality 14, 768 (2002).

11. L. D. Barron, Molecular Light Scattering and Optical Activity (Cambridge University Press Cambridge, UK, 2004).

12. D. S. Bradshaw and D. L. Andrews, New J. Phys. 16, 103021 (2014).

13. K. A. Forbes and D. L. Andrews, Phys. Rev. A 91, 053824 (2015).

14. F. Araoka, T. Verbiest, K. Clays, and A. Persoons, Phys. Rev. A 71, 055401 (2005).

15. W. Löffler, D. Broer, and J. Woerdman, Phys. Rev. A 83 065801 (2011).

16. E. A. Power and T. Thirunamachandran, Am. J. Phys. 46, 370 (1978).

17. L. Allen, M. W. Beijersbergen, R. J. C. Spreeuw, and J. P. Woerdman, Phys. Rev. A 45, 8185 (1992).

18. S. M. Barnett, M. Babiker, and M. J. Padgett, ed., Optical Orbital Angular Momentum (The Royal Society, 2017).

19. G. Nienhuis, "Angular Momentum and Vortices in Optics," in Structured Light and Its Applications (Academic Press, Burlington, 2008).

20. D. L. Andrews and T. Thirunamachandran, J. Chem. Phys. 67, 5026 (1977).

21. E. Bendau, L. Zhang, R. Gozali, S. Ashrafi, and R. R. Alfano, in Complex Light and Optical Forces XI, Proc. SPIE 10120, 1012004 (2017).

22. R. W. Boyd, Nonlinear Optics (Academic Press, New York, 2003).

$23 . \quad$ Y. R. Shen, JOSA B 28, A56 (2011)

24. M. Lemeshko, R. V. Krems, J. M. Doyle, and S. Kais, Mol. Phys. 111, 1648 (2013).

25. W. R. Mason, Magnetic circular dichroism spectroscopy (John Wiley \& Sons, 2007).

26. V. Lembessis and M. Babiker, Phys. Rev. Lett. 110, 083002 (2013).

27. A. Afanasev, C. E. Carlson, and A. Mukherjee, J. Opt 18, 074013 (2016).

28. T. Wu, W. Zhang, R. Wang, and X. Zhang, Nanoscale 9, 5110 (2017).

29. M. G. Mandujano and J. A. Maytorena, Phys. Rev. A 88 023811 (2013).

30. D. Gao, W. Ding, M. Nieto-Vesperinas, X. Ding, M. Rahman, T. Zhang, C. Lim, and C.-W. Qiu, Light Sci Appl. 6, e17039 (2017).

31. N. Berova, P. L. Polavarapu, K. Nakanishi, and R. W. Woody, Comprehensive chiroptical spectroscopy, applications in stereochemical analysis of synthetic compounds, natural products, and biomolecules (John Wiley \& Sons, 2012).

32. A. Canaguier-Durand, J. A. Hutchison, C. Genet, and T. W. Ebbesen, New J. Phys. 15, 123037 (2013).

33. D. S. Bradshaw and D. L. Andrews, Opt. Lett. 40, 677 (2015). 
[19] G. Nienhuis, in Structured Light and Its Applications (Academic Press, Burlington, 2008).

[20] D. L. Andrews and T. Thirunamachandran, On threedimensional rotational averages, J. Chem. Phys. 67, 5026 (1977).

[1] D. L. Andrews and M. Babiker, The Angular Momentum of Light (Cambridge University Press, Cambridge, UK, 2013).

[2] G. Gibson, J. Courtial, M. J. Padgett, M. Vasnetsov, V. Pas'ko, S. M. Barnett, and S. Franke-Arnold, Free-space information transfer using light beams carrying orbital angular momentum, Opt. Express 12, 5448 (2004).

[3] D. L. Andrews, L. C. Dávila Romero, and M. Babiker, On optical vortex interactions with chiral matter, Opt. Commun. 237, 133 (2004).

[4] X. Zambrana-Puyalto, X. Vidal, and G. Molina-Terriza, Angular momentum-induced circular dichroism in non-chiral nanostructures, Nat. Commun. 5, 4922 (2014).

[5] W. Brullot, M. K. Vanbel, T. Swusten, and T. Verbiest, Resolving enantiomers using the optical angular momentum of twisted light, Science Advances 2, e1501349 (2016).

[6] D. J. Caldwell and H. Eyring, The Theory of Optical Activity (Wiley-Interscience, New York, 1971).

[7] S. F. Mason, Molecular optical activity and the chiral discriminations (Cambridge University Press, 1982).

[8] D. P. Craig and T. Thirunamachandran, Molecular Quantum Electrodynamics: An Introduction to Radiation-Molecule Interactions (Dover Publications, Mineola, NY, 1998).

[9] M. Nieto-Vesperinas, Chiral optical fields: a unified formulation of helicity scattered from particles and dichroism enhancement, Phil. Trans. R. Soc. A 375, 20160314 (2017).

[10] P. L. Polavarapu, Optical rotation: Recent advances in determining the absolute configuration, Chirality 14 , 768 (2002).

[11] L. D. Barron, Molecular Light Scattering and Optical Activity (Cambridge University Press Cambridge, UK, 2004).

[12] D. S. Bradshaw and D. L. Andrews, Chiral discrimination in optical trapping and manipulation, New J. Phys. 16, 103021 (2014).

[13] K. A. Forbes and D. L. Andrews, Chiral discrimination in optical binding, Phys. Rev. A 91, 053824 (2015).

[14] F. Araoka, T. Verbiest, K. Clays, and A. Persoons, Interactions of twisted light with chiral molecules: an experimental investigation, Phys. Rev. A 71, 055401 (2005).

[15] W. Löffler, D. Broer, and J. Woerdman, Circular dichroism of cholesteric polymers and the orbital angular momentum of light, Phys. Rev. A 83, 065801 (2011).

[16] E. A. Power and T. Thirunamachandran, On the nature of Hamiltonian for interaction of radiation with atoms and molecules: (e/mc)p.A, $-\mu$.E, and all that, Am. J. Phys. 46, 370 (1978).

[17] L. Allen, M. W. Beijersbergen, R. J. C. Spreeuw, and J. P. Woerdman, Orbital angular momentum of light and the transformation of Laguerre-Gaussian laser modes, Phys. Rev. A 45, 8185 (1992).

[18] S. M. Barnett, M. Babiker, and M. J. Padgett, ed., Optical Orbital Angular Momentum (The Royal Society, 2017).

[21] E. Bendau, L. Zhang, R. Gozali, S. Ashrafi, and R. R. Alfano, in Complex Light and Optical Forces XI, Proc. SPIE 10120, 1012004 (2017).

[22] R. W. Boyd, Nonlinear Optics (Academic Press, New York, 2003).

[23] Y. R. Shen, Surface nonlinear optics, JOSA B 28, A56 (2011).

[24] M. Lemeshko, R. V. Krems, J. M. Doyle, and S. Kais, Manipulation of molecules with electromagnetic fields, Mol. Phys. 111, 1648 (2013).

[25] W. R. Mason, Magnetic circular dichroism spectroscopy (John Wiley \& Sons, 2007).

[26] V. Lembessis and M. Babiker, Enhanced quadrupole effects for atoms in optical vortices, Phys. Rev. Lett. 110, 083002 (2013).

[27] A. Afanasev, C. E. Carlson, and A. Mukherjee, Highmultipole excitations of hydrogen-like atoms by twisted photons near a phase singularity, J. Opt 18, 074013 (2016).

[28] T. Wu, W. Zhang, R. Wang, and X. Zhang, A giant chiroptical effect caused by the electric quadrupole, Nanoscale 9, 5110 (2017).

[29] M. G. Mandujano and J. A. Maytorena, Quadrupolar second-harmonic generation by helical beams and vectorial vortices with radial or azimuthal polarization, Phys. Rev. A 88, 023811 (2013).

[30] D. Gao, W. Ding, M. Nieto-Vesperinas, X. Ding, M. Rahman, T. Zhang, C. Lim, and C.-W. Qiu, Optical manipulation from the microscale to the nanoscale: fundamentals, advances and prospects, Light Sci Appl. 6, e17039 (2017).

[31] N. Berova, P. L. Polavarapu, K. Nakanishi, and R. W. Woody, Comprehensive chiroptical spectroscopy, applications in stereochemical analysis of synthetic compounds, natural products, and biomolecules (John Wiley \& Sons, 2012), Vol. 2.

[32] A. Canaguier-Durand, J. A. Hutchison, C. Genet, and T. W. Ebbesen, Mechanical separation of chiral dipoles by chiral light, New J. Phys. 15, 123037 (2013).

[33] D. S. Bradshaw and D. L. Andrews, Laser optical separation of chiral molecules, Opt. Lett. 40, 677 (2015). 